Pacific Journal of Mathematics

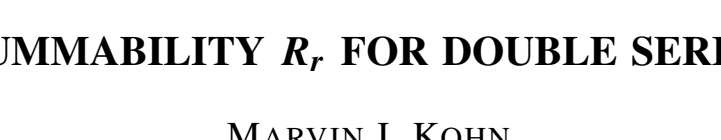




\title{
SUMMABILITY $R_{r}$ FOR DOUBLE SERIES
}

\author{
M. J. KoHN
}

Let $r$ be a positive integer. $A$ trigonometric series $T$ of a single variable is said to be summable $R_{r}$ at $\theta_{0}$ if the series obtained by $r$ times formally integrating $T$ has an $r$ th symmetric derivative at $\theta_{o}$. For even values of $r$, summability $R_{r}$ has been applied to double trigonometric series. We study here summability $R_{r}$, for odd values of $r$, for double trigonometric series. We obtain a connection between Bochner-Riesz summable series and series which are summable $R_{r}$.

1. Let

$$
\sum_{-\infty}^{\infty} c_{n} e^{i n \theta}
$$

be a trigonometric series of a single variable. Let $r$ be a positive integer. Suppose the series obtained by formally integrating (1.1) $r$ times

$$
c_{o} \frac{\theta^{r}}{r !}+\sum_{n \neq 0} \frac{c_{n}}{(i n)^{r}} e^{i n \theta}
$$

converges to a function $F(\theta)$ in a neighborhood of $\theta_{0} \in(0,2 \pi)$. We will say that the series (1.1) is at $\theta_{0}$ summable by the method $R_{r}$ to sum $s$ if $F(\theta)$ has at $\theta_{0}$ an $r$ th symmetric derivative with value $s$. That is, if $r$ is even,

$$
\frac{1}{2}\left\{F\left(\theta_{o}+t\right)+F\left(\theta_{o}-t\right)\right\}=a_{o}+\frac{a_{2}}{2 !} t^{2}+\cdots+\frac{s}{r !} t^{r}+o\left(t^{r}\right)
$$

as $t \rightarrow 0$, and if $r$ is odd,

$$
\frac{1}{2}\left\{F\left(\theta_{o}+t\right)-F\left(\theta_{o}-t\right)\right\}=a_{1} t+\frac{a_{3}}{3 !} t^{3}+\cdots+\frac{s}{r !} t^{r}+o\left(t^{r}\right),
$$

as $t \rightarrow 0$.

The following result, see [8], p. 66, establishes a connection between summability $(C, \alpha)$ and summability $R_{r}$ for trigonometric series.

THEOREM A. Let $\alpha>-1$ and assume the series (1.1) is summable $(C, \alpha)$ at $\theta_{0}$ to sum s. Let $r$ be an integer with $r>\alpha+1$, and suppose the series (1.2) converges in a neighborhood of $\theta_{0}$. Then the series (1.1) is summable $R_{r}$ to $s$.

2. In two variables we will denote points $x \in E_{2}$ by $x=\left(x_{1}, x_{2}\right)=$ 
$t e^{2 \theta}$ and integral lattice points by $n=\left(n_{1}, n_{2}\right)$. We write

$$
|x|=\sqrt{x_{1}^{2}+x_{2}^{2}} \text {. }
$$

We will say a double trigonometric series

$$
T: \sum_{n \in Z_{2}} c_{n} e^{i n \cdot x}
$$

is Bochner-Riesz summable of order $\alpha$ at $\varkappa_{o}$ to sum $s_{o}$ if

$$
\lim _{R \rightarrow \infty} \sum_{\mid n i<R}\left(1-\left(\frac{|n|}{R}\right)^{2}\right)^{\alpha} c_{n} e^{i n \cdot x_{o}}=s_{o} .
$$

Suppose $r$ is an even number, $r=2 s$. A two dimensional analogue of summability $R_{r}$ is given as follows, see [7], [4].

Definition. Let $F(x)$ be defined in a neighborhood of $x_{0} \in E_{2}$. $F$ has at $x_{0}$ a sth generalized Laplacian equal to $s_{0}$ if $F$ is integrable on each circle $\left|x-x_{0}\right|=t$ and

$$
\frac{1}{2 \pi} \int_{0}^{2 \pi} F\left(x_{0}+t e^{\imath \theta}\right) d \theta=a_{0}+\frac{a_{2} t^{2}}{(2 !)^{2}}+\cdots+\frac{s_{0} t^{2 s}}{\left(2^{s} s !\right)^{2}}+o\left(t^{2 s}\right)
$$

as $t \rightarrow 0$.

THEOREM B. Let the series $T$ of (2.1) be Bochner-Riesz-m summable at $x_{0}$ to sum $s_{o}$, where $m$ is a nonnegative integer, and suppose the coefficients of $T$ satisfy

$$
\sum_{n \in Z_{2}}|n|^{-3+\varepsilon}\left|c_{n}\right|^{2}<\infty
$$

for some $\varepsilon>0$. Let $r=2 s$ be an even integer with $r \geqq m+2$. Set

$$
F(x)=\frac{c_{o}\left(x_{1}+x_{2}\right)^{2 s}}{2^{s}(2 s) !}+(-1)^{s} \sum_{n \neq 0} \frac{c_{n}}{|n|^{2 s}} e^{2 n \cdot x} .
$$

Then the generalized sth Laplacian of $F(x)$ exists at $x_{0}$ and is equal to $s_{0}$.

That is, if the series (2.1) is Bochner-Riesz-m summable to $s_{0}$ and $r$ is an even number with $r \geqq m+2$, then the series is also summable $R_{r}$ to sum $s_{0}$.

3. The purpose of this paper is to derive a connection between Bochner-Riesz summability and summability $R_{r}$, for odd values of $r$. We use the following definition, from [5]. This definition extends the formula of (1.4) to two dimensions in a manner analogous to the extension of (1.3) to two variables by (2.2). 
DEFINITION. Let $r=2 s+1$ be an odd positive integer. Let $L(x)$ be a function defined in a neighborhood of $x_{0} \in E_{2}$. We will say $L(x)$ has at $x_{0}$ a generalized symmetric derivative of order $r$ with value $s_{o}$ if $L$ is integrable on each circle $\left|x-x_{0}\right|=t$, for $t$ small, and if

$$
\begin{aligned}
& \frac{1}{2 \pi} \int_{0}^{2 \pi} L\left(x_{o}+t e^{i \theta}\right)(\cos \theta+\sin \theta) d \theta \\
& \quad=a_{1} t+a_{3} t^{3}+\cdots+\frac{s_{o}}{2^{2 s+1} s !(s+1) !} t^{2 s+1}+o\left(t^{2 s+1}\right)
\end{aligned}
$$

as $t \rightarrow 0$.

We are able to obtain the following results which, for odd values of $r$, form a two dimensional version of Theorem A. We begin with the case of double trigonometric series which are Bochner-Riesz summable of integral order, since the statement and proof of our results are much simpler in this case.

Theorem 1. Let $m$ be a nonnegative integer. Suppose

$$
T: \sum_{n \in Z_{2}} c_{n} e^{i n \cdot x}
$$

is Bochner-Riesz-m summable at $x_{o}$ to finite sum $s_{o}$. Let $r=2 s+1$ be an odd integer such that $r \geqq m+1$. Suppose the coefficients of $T$ satisfy

$$
\sum_{n_{1}+n_{2}=0}|n|^{-2 r+3+\varepsilon}\left|c_{n}\right|^{2}+\sum_{n_{1}+n_{2} \neq 0}\left(n_{1}+n_{2}\right)^{-2}|n|^{-2 r+3+\varepsilon}\left|c_{n}\right|^{2}<\infty
$$

for some $\varepsilon>0$. Then the series

$$
\begin{aligned}
\frac{c_{0}\left(x_{1}+x_{2}\right)^{r}}{(r) !(2 r) ! 2^{s+1}} & +\frac{1}{2}\left(x_{1}+x_{2}\right) \sum_{n_{1}+n_{2}=0}^{\prime} \frac{c_{n}}{|n|^{2 s}} e^{i n \cdot x} \\
& +\sum_{n_{1}+n_{2} \neq 0} \frac{-i c_{n}}{\left(n_{1}+n_{2}\right)|n|^{2 s}} e^{i n \cdot x}
\end{aligned}
$$

converges spherically to a function $L(x)$ which has at $x_{o}$ a generalized symmetric derivative of order $r$ with value $s_{0}$.

We are able to extend Theorem 1 to include some, but not all, fractional orders of Bochner-Riesz summability. Let $\beta$ be a nonnegative real number. We denote by $[\beta]$ the largest integer $\leqq \beta$ and by $\langle\beta\rangle$ the fractional part of $\beta,\langle\beta\rangle=\beta-[\beta]$.

THeOREM 2. Let $\beta$ be a nonnegative real number with $\langle\beta\rangle<$ 1/2. Suppose the series (3.2) is summable Bochner-Riesz- $\beta$ to finite sum $s_{0}$. Let $r=2 s+1$ be an odd integer with $r \geqq[\beta]+1$. Suppose the coefficients of the series (3.2) satisfy formula (3.3) for some $\varepsilon>0$. 
Then the conclusion of Theorem 1 still holds.

In particular, in the two dimensional case, Bochner-Riesz summability of order $\beta$, for $\beta<1 / 2$, is enough to imply summability $R_{1}$ (which is Lebesgue summability).

4. Although Theorem 1 is a special case of Theorem 2, we give its proof separately, since its proof is much easier than that of Theorem 2. We will assume, as we may, that $c_{0}=0, x_{0}=0$, and $s_{o}=0$. We set

$$
S_{R}=S_{R}(0)=\sum_{|n|<R} c_{n},
$$

and for $\eta>0$

$$
S_{R}^{\eta}=\frac{1}{\Gamma(\eta)} \int_{0}^{R}(R-u)^{\eta-1} S_{u} d u
$$

Note that $S_{R}^{\eta}$, as a function of $R$, is the fractional integral of order $\eta$ of $f(R)=S_{R}$, see [6].

Hardy, see [2], has shown that a series $\sum c_{n}$ is Bochner-Riesz- $\eta$ summable to 0 if and only if

$$
\sum_{|n|<R} c_{n}\left(1-\frac{|n|}{R}\right)^{\eta} \rightarrow 0
$$

as $R \rightarrow \infty$. Thus, for the proof of Theorem 1 we may assume

$$
S_{R}^{m}=o\left(R^{m}\right)
$$

as $R \rightarrow \infty$.

We will need the following lemmas. The first lemma has been adapted from [7].

Lemma 1. Suppose $\sum_{n \in z_{2}} c_{n} e^{i n \cdot x}$ is Bochner-Riesz- $(m+1)$ summable to 0 at $x=0$, and suppose the coefficients $c_{n}$ satisfy condition (3.3) of Theorem 1, with $r \geqq m+1$. Then

$$
S_{R}^{k}=o\left(R^{r+1 / 2}\right),
$$

as $R \rightarrow \infty$, for $k=0,1, \cdots, m+1$.

Proof. We first note that for $n_{1}+n_{2} \neq 0$,

$$
\begin{aligned}
\sum_{n_{1}+n_{2} \neq 0} & \left(n_{1}+n_{2}\right)^{-2}|n|^{-2 r+3+\varepsilon}\left|c_{n}\right|^{2} \\
& \geqq \frac{1}{4} \sum_{n_{1}+n_{2} \neq 0}|n|^{-2}|n|^{-2 r+3+\varepsilon}\left|c_{n}\right|^{2} \\
& =\frac{1}{4} \sum_{n_{1}+n_{2} \neq 0}|n|^{-2 r+1+\varepsilon}\left|c_{n}\right|^{2} .
\end{aligned}
$$


Thus, from (3.3),

$$
\sum_{n_{1}+n_{2} \neq 0}|n|^{-2 r+1+\varepsilon}\left|c_{n}\right|^{2}<\infty
$$

and therefore

$$
\sum_{n \in Z_{2}}|n|^{-2 r+1+\varepsilon}\left|c_{n}\right|^{2}<\infty .
$$

Using Schwartz's inequality,

$$
\begin{aligned}
\sum_{|n|<R}\left|c_{n}\right| & =\sum_{|n|<R}\left(|n|^{1 / 2(-2 r+1+\varepsilon)}\left|c_{n}\right|\right)\left(|n|^{-1 / 2(-2 r+1+\varepsilon)}\right) \\
& \leqq\left(\sum_{n \in Z_{2}}|n|^{-2 r+1+\varepsilon}\left|c_{n}\right|^{2}\right)^{1 / 2}\left(\sum_{|n|<R}|n|^{2 r-1-\varepsilon}\right)^{1 / 2} \\
& =C \cdot\left(R^{2 r+1-\varepsilon}\right)^{1 / 2} \\
& =o\left(R^{r+1 / 2}\right)
\end{aligned}
$$

as $R \rightarrow \infty$.

Now fix an integer $j$.

$$
\begin{gathered}
\sum_{|i|<R} c_{i}(R-|i|+j)^{m+1}=\sum_{|i|<R+j} c_{i}(R-|i|+j)^{m+1} \\
-\sum_{R \leqq|i|<R+j} c_{i}(R-|i|+j)^{m+1} .
\end{gathered}
$$

Since $\sum c_{n} e^{i n \cdot x}$ is Bochner-Riesz- $(m+1)$ summable to 0 at 0 ,

$$
\sum_{|i|<R+j} c_{i}(R-|i|+j)^{m+1}=o\left(R^{m+1}\right)
$$

as $R \rightarrow \infty$.

$$
\sum_{R \leqq|i|<R+j} c_{i}(R-|i|+j)^{m+1}=o\left(R^{r+1 / 2}\right),
$$

because of (4.4). Thus,

$$
\begin{aligned}
\sum_{|i|<R} c_{i}(R-|i|+j)^{m+1} & =o\left(R^{m+1}\right)+o\left(R^{r+1 / 2}\right) \\
& =o\left(R^{r+1 / 2}\right),
\end{aligned}
$$

as $R \rightarrow \infty$.

We next use the fact, see [7], that there are number $C_{j k}$, for $j=1, \cdots, m+2, k=0, \cdots, m+1$ such that for all complex numbers $z$,

$$
\sum_{j=1}^{m+2} C_{j k}(z+j)^{m+1}=z^{k}
$$

Thus, for $0 \leqq k \leqq m+1$,

$$
\begin{aligned}
S_{R}^{k} & =\frac{1}{k !} \sum_{|i|<R} c_{i}(R-|i|)^{k} \\
& =\frac{1}{k !} \sum_{|i|<R} c_{i} \sum_{j=1}^{m+2} C_{j k}(R-|i|+j)^{m+1}
\end{aligned}
$$




$$
\begin{aligned}
& =\sum_{j=1}^{m+2} \frac{1}{k !} C_{j k} \sum_{|i|<R} c_{i}(R-|i|+j)^{m+1} \\
& =\sum_{j=1}^{m+2} \frac{1}{k !} C_{j k} o\left(R^{r+1 / 2}\right) \\
& =o\left(R^{r+1 / 2}\right),
\end{aligned}
$$

by (4.5). This proves Lemma 1.

LEMma 2. Let $x=\left(x_{1}, x_{2}\right)=t e^{i \theta} \in E_{2}$ and $n=\left(n_{1}, n_{2}\right) \in \boldsymbol{Z}_{2}$, with $|n| \neq 0 . \quad$ Define

$$
g_{n}(x)=\left\{\begin{array}{lll}
\frac{1}{2}\left(x_{1}+x_{2}\right) e^{i n \cdot x} & \text { if } & n_{1}+n_{2}=0 \\
\frac{-i e^{i n \cdot x}}{n_{1}+n_{2}} & \text { if } & n_{1}+n_{2} \neq 0 .
\end{array}\right.
$$

Then

$$
\frac{1}{2 \pi} \int_{0}^{2 \pi} g_{n}\left(t e^{i \theta}\right)(\cos \theta+\sin \theta) d \theta=\frac{J_{1}(|n| t)}{|n|},
$$

where $J_{1}(z)$ is the Bessel function of the first kind of order 1.

Proof. This is the lemma from [5].

5. Proof of Theorem 1. Let

$$
T_{R}(x)=\sum_{\substack{|n|<R \\ n_{1}+n_{2}=0}} \frac{1}{2}\left(x_{1}+x_{2}\right) \frac{c_{n}}{|n|^{2 s}} e^{i n \cdot x}+\sum_{\substack{|n|<R \\ n_{1}+n_{2} \neq 0}} \frac{-i c_{n}}{\left(n_{1}+n_{2}\right)|n|^{2 s}} e^{i n \cdot x} .
$$

The hypothesis (3.3) insures that

$$
L(x)=\lim _{R \rightarrow \infty} T_{R}(x)
$$

exists a.e. on each circle $|x|=t$, see [3], Theorem 1. Also, by Theorem 2 of [3],

$$
\int_{0}^{2 \pi} \sup _{R}\left|T_{R}\left(t e^{i \theta}\right)\right| d \theta<\infty,
$$

so, using Lebesgue's Dominated Convergence Theorem,

$$
\begin{aligned}
\frac{1}{2 \pi} \int_{0}^{2 \pi} & L\left(t e^{i \theta}\right)(\cos \theta+\sin \theta) d \theta \\
& =\lim _{R \rightarrow \infty} \frac{1}{2 \pi} \int_{0}^{2 \pi} T_{R}\left(t e^{i \theta}\right)(\cos \theta+\sin \theta) d \theta \\
& =\lim _{R \rightarrow \infty} \sum_{|n|<R} \frac{c_{n}}{|n|^{2 s}} \frac{1}{2 \pi} \int_{0}^{2 \pi} g_{n}\left(t e^{i \theta}\right)(\cos \theta+\sin \theta) d \theta
\end{aligned}
$$


where $g_{n}(x)$ is defined by (4.6). Using Lemma 2 we get

$$
\begin{aligned}
\frac{1}{2 \pi} \int_{0}^{2 \pi} & L\left(t e^{i \theta}\right)(\cos \theta+\sin \theta) d \theta \\
& =\lim _{R \rightarrow \infty} \sum_{|n|<R} \frac{c_{n}}{|n|^{2 s}} \frac{J_{1}(|n| t)}{|n|} \\
& =\lim _{R \rightarrow \infty} \sum_{|n|<R} c_{n} \frac{J_{1}(|n| t)}{|n|^{r}} \\
& =t^{r} \lim _{R \rightarrow \infty} \sum_{|n|<R} c_{n} \gamma(|n| t),
\end{aligned}
$$

where $\gamma(t)=z^{-r} J_{1}(z)$.

We express the last sum as an integral and integrate by parts $m+1$ times.

$$
\begin{aligned}
& \sum_{|n|<R} c_{n} \gamma(|n| t)=S_{R} \gamma(R t)-\int_{0}^{R} S_{u} \frac{d}{d u} \gamma(u t) d u \\
& =S_{R} \gamma(R t)-S_{R}^{1} \frac{d}{d R} \gamma(R t)+\int_{0}^{R} S_{u}^{1} \frac{d^{2}}{d u^{2}} \gamma(u t) d u
\end{aligned}
$$

$$
\begin{aligned}
= & S_{R} \gamma(R t)-S_{R}^{1} \frac{d}{d R} \gamma(R t)+\cdots+(-1)^{m} S_{R}^{m} \frac{d^{m}}{d R^{m}} \gamma(R t) \\
& +(-1)^{m+1} \int_{0}^{R} S_{u}^{m} \frac{d^{m+1}}{d u^{m+1}} \gamma(u t) d u .
\end{aligned}
$$

From Lemma 1,

$$
S_{R}^{k}=o\left(R^{r+1 / 2}\right) \text { for } k=0, \cdots, m .
$$

Repeatedly using the relations from [1],

$$
\frac{d}{d z}\left(z^{-n} J_{n}(z)\right)=z^{-n} J_{n+1}(z)
$$

and

$$
J_{\nu}(z)=o\left(z^{-1 / 2}\right)
$$

as $z \rightarrow \infty$, we get

$$
\frac{d^{k}}{d z_{k}} \gamma(z)=o\left(z^{-r-1 / 2}\right)
$$

as $z \rightarrow \infty$. So, for $k=0, \cdots, m$

$$
\begin{aligned}
S_{R}^{k} \frac{d^{k}}{d R^{k}} \gamma(R t) & =o\left(R^{r+1 / 2}\right) o\left(R^{-r-1 / 2}\right) \\
& =o(1),
\end{aligned}
$$


as $R \rightarrow \infty$. Thus, returning to (5.2),

$$
\lim _{R \rightarrow \infty} \sum_{|n|<R} c_{n} \gamma(|n| t)=(-1)^{m+1} \int_{0}^{\infty} S_{u}^{m} \frac{d^{m+1}}{d u^{m+1}} \gamma(u t) d u,
$$

and (5.1) becomes,

$$
\begin{aligned}
& \frac{1}{2 \pi} \int_{0}^{2 \pi} L\left(t e^{i \theta}\right)(\cos \theta+\sin \theta) d \theta \\
& \quad=t^{r} \lim _{R \rightarrow \infty} \sum_{|n|<R} c_{n} \gamma(|n| t) \\
& \quad=t^{r}(-1)^{m+1} \int_{0}^{\infty} S_{u}^{m} \frac{d^{m+1}}{d u^{m+1}} \gamma(u t) d u .
\end{aligned}
$$

Now we make use of the series expansion for $J_{1}(z),[1]$, p. 4 .

$$
\begin{aligned}
J_{1}(z) & =\sum_{k=0}^{\infty} \frac{(-1)^{k}\left(\frac{1}{2} z\right)^{1+2 k}}{k !(k+1) !} \\
& =a_{1} z+a_{3} z^{3}+\cdots .
\end{aligned}
$$

Then,

$$
\begin{aligned}
\gamma(z) & =z^{-r} J_{1}(z) \\
& =z^{-r}\left(a_{1} z+a_{3} z^{3}+\cdots+a_{r-2} z^{r-2}+a_{r} z^{r}+\cdots\right) .
\end{aligned}
$$

We define a polynomial $P(z)$ as follows. If $r=1$, let $P(z) \equiv 0$. Otherwise, let

$$
P(z)=a_{1} z+a_{3} z^{3}+\cdots+a_{r-2} z^{r-2}
$$

where the $a_{i}$ 's are given by (5.7). Now we let

$$
\lambda(z)=\gamma(z)-z^{-r} P(z) \text {. }
$$

Then $\lambda(z)$ is an entire function in the plane and

$$
\gamma(z)=z^{-r} P(z)+\lambda(z) \text {. }
$$

Returning to (5.6),

$$
\begin{aligned}
\frac{1}{2 \pi} \int_{0}^{2 \pi} & L\left(t e^{i \theta}\right)(\cos \theta+\sin \theta) d \theta \\
= & t^{r}(-1)^{m+1} \int_{0}^{\infty} S_{u}^{m} \frac{d^{m+1}}{d u^{m+1}} \gamma(u t) d u \\
= & t^{r}(-1)^{m+1} \int_{0}^{\infty} S_{u}^{m} \frac{d^{m+1}}{d u^{m+1}}\left\{(u t)^{-r} P(u t)+\lambda(u t)\right\} d u \\
= & t^{r}(-1)^{m+1} \int_{0}^{\infty} S_{u}^{m} \frac{d^{m+1}}{d u^{m+1}}\left\{(u t)^{-r} P(u t)\right\} d u \\
& +t^{r}(-1)^{m+1} \int_{0}^{\infty} S_{u}^{m} \frac{d^{m+1}}{d u^{m+1}} \lambda(u t) d u \\
= & A+t^{r} B(t) .
\end{aligned}
$$


Since $c_{o}=0$, therefore $S_{u}^{m}=0$ for $0 \leqq u<1$. Thus we may replace the interval of integration of the integral involving $A$ by the interval $(1 / 2, \infty)$.

$$
\begin{aligned}
A & =t^{r}(-1)^{m+1} \int_{1 / 2}^{\infty} S_{u}^{m} \frac{d^{m+1}}{d u^{m+1}}\left\{(u t)^{-r} P(u t)\right\} d u \\
& =t^{r}(-1)^{m+1} \int_{1 / 2}^{\infty} S_{u}^{m} \frac{d^{m+1}}{d u^{m+1}}\left(\sum_{\substack{k=1 \\
k \text { odd }}}^{r-2} a_{k}(u t)^{k-r}\right) d u \\
& =\sum_{\substack{k=1 \\
k \text { odd }}}^{r-2} t^{r+k-r} a_{k}(-1)^{m+1} \int_{1 / 2}^{\infty} S_{u}^{m} \frac{d^{m+1}}{d u^{m+1}} u^{k-r} d u \\
& =\sum_{\substack{k=1 \\
k=1}}^{r-2} t^{k} a_{k}(-1)^{m+1} \int_{1 / 2}^{\infty} o\left(u^{m}\right) O\left(u^{k-r-m-1}\right) d u \\
& =\sum_{\substack{k=1 \\
k \text { odd }}}^{r-2} t^{k} a_{k}(-1)^{m+1} \int_{1 / 2}^{\infty} o\left(u^{k-r-1}\right) d u \\
& =\sum_{\substack{k=1 \\
k=1}}^{r-2} b_{k} t^{k} .
\end{aligned}
$$

Returning to (5.9),

$$
\begin{aligned}
& \frac{1}{2 \pi} \int_{0}^{2 \pi} L\left(t e^{i \theta}\right)(\cos \theta+\sin \theta) d \theta \\
& \quad=A+t^{r} B(t) \\
& \quad=b_{1} t+b_{3} t^{3}+\cdots+b_{r-2} t^{r-2}+0 \cdot t^{r}+t^{r} B(t) .
\end{aligned}
$$

The proof of Theorem 1 will be complete when we establish $B(t) \rightarrow 0$ as $t \rightarrow 0$.

$$
\begin{aligned}
B(t) & =(-1)^{m+1} \int_{0}^{\infty} S_{u}^{m} \frac{d^{m+1}}{d u^{m+1}} \lambda(u t) d u \\
& =\int_{0}^{1 / t}+\int_{1 / t}^{\infty} \\
& =B_{1}(t)+B_{2}(t) .
\end{aligned}
$$

To estimate $B_{1}(t)$ we use the fact that $\lambda(z)$ is entire, so for $|z| \leqq 1$,

$$
\left|\frac{d^{k}}{d z^{k}} \lambda(z)\right|<K
$$

Since $|u t| \leqq 1$ in the interval of integration involving $B_{1}(t)$,

$$
\left|\frac{d^{m+1}}{d u^{m+1}} \lambda(u t)\right| \leqq t^{m+1} K
$$

in this interval. 


$$
\begin{aligned}
B_{1}(t) & =(-1)^{m+1} \int_{0}^{1 / t} o\left(u^{m}\right) t^{m+1} K d u \\
& =o\left(t^{m+1}\right) \int_{0}^{1 / t} u^{m} d u \\
& =o\left(t^{m+1}\right)\left(\frac{1}{t}\right)^{m+1} \\
& =o(1)
\end{aligned}
$$

as $t \rightarrow 0$.

For the estimate of $B_{2}(t)$ we use the decomposition

$$
\lambda(z)=\gamma(z)-z^{-r} P(z)
$$

Clearly, as $z \rightarrow \infty$

$$
\frac{d^{m+1}}{d z^{m+1}} z^{-r} P(z)=O\left(z^{-m-3}\right)
$$

and by (5.4),

$$
\frac{d^{m+1}}{d z^{m+1}} \gamma(z)=O\left(z^{-r-1 / 2}\right)
$$

Thus, for $z \rightarrow \infty$

$$
\frac{d^{m+1}}{d z^{m+1}} \lambda(z)=O\left(z^{-r-1 / 2}\right)
$$

and

$$
\begin{aligned}
B_{2}(t) & =(-1)^{m+1} \int_{1 / t}^{\infty} S_{u}^{m} \frac{d^{m+1}}{d u^{m+1}} \lambda(u t) d u \\
& =(-1)^{m+1} \int_{1 / t}^{\infty} o\left(u^{m}\right) t^{m+1} O(u t)^{-r-1 / 2} d u \\
& =o\left(t^{m+1-r-1 / 2}\right) \int_{1 / t}^{\infty} o(u)^{m-r-1 / 2} d u \\
& =o\left(t^{m-r+1 / 2}\right) o\left(\frac{1}{t}\right)^{m-r+1 / 2} \\
& =o(1) .
\end{aligned}
$$

(Note we needed $m-r-1 / 2<-1$ to perform the last integration.) Thus $B_{2}(t) \rightarrow 0$ as $t \rightarrow 0$, and returning to (5.10), the proof of Theorem 1 is complete.

6. Proof of Theorem 2. We may assume that the fractional part of $\beta$ is not zero. Otherwise Theorem 2 reduces to Theorem 1 . Write $\beta=m+\alpha$, where $m$ is an integer and $0<\alpha<1 / 2$.

We again assume $c_{o}=0, x_{o}=0, s_{o}=0$. We proceed as in the beginning of the proof of Theorem 1 . 


$$
\begin{array}{r}
\frac{1}{2 \pi} \int_{0}^{2 \pi} L\left(t e^{i \theta}\right)(\cos \theta+\sin \theta) d \theta \\
\quad=t^{r} \lim _{R \rightarrow \infty} \sum_{|n|<R} c_{n} \gamma(|n| t),
\end{array}
$$

with $\gamma(z)=z^{-r} J_{1}(z)$.

As in the proof of Theorem 1 we integrate the last sum by parts. We now integrate by parts $m+2$ times.

$$
\begin{aligned}
\sum_{|n|<R} c_{n} \gamma(|n| t)= & S_{R} \gamma(R t)-\int_{0}^{R} S_{u} \frac{d}{d u} \gamma(u t) d u \\
\vdots & =S_{R} \gamma(R t)-S_{R}^{1} \frac{d}{d R} \gamma(R t)+\cdots+(-1)^{m+1} S_{R}^{m+1} \frac{d^{m+1}}{d R^{m+1}} \gamma(R t) \\
& +(-1)^{m+2} \int_{0}^{R} S_{u}^{m+1} \frac{d^{m+2}}{d u^{m+2}} \gamma(u t) d u .
\end{aligned}
$$

We are now assuming the series (3.1) is summable BochnerRiesz- $\beta$ to 0 at $x_{o}=0$, so it is also summable Bochner-Riesz- $(m+1)$ to 0 at $x_{o}=0$. Therefore we may again apply Lemma 1. For $0 \leqq k \leqq m+1$,

$$
\begin{aligned}
S_{R}^{k} \frac{d^{k}}{d R^{k}} \gamma(R t) & =o\left(R^{r+1 / 2}\right) O\left(R^{-r-1 / 2}\right) \\
& =o(1)
\end{aligned}
$$

as $R \rightarrow \infty$, so

$$
\begin{aligned}
& \frac{1}{2 \pi} \int_{0}^{2 \pi} L\left(t e^{i \theta}\right)(\cos \theta+\sin \theta) d \theta \\
& \quad=t^{r} \lim _{R \rightarrow \infty} \sum_{|n|<R} c_{n} \gamma(|n| t) \\
& \quad=t^{r}(-1)^{m} \int_{0}^{\infty} S_{u}^{m+1} \frac{d^{m+2}}{d u^{m+2}} \gamma(u t) d u .
\end{aligned}
$$

We define $P(z)$ and $\lambda(z)$ as in the proof of Theorem 1:

$$
P(z)=\left\{\begin{array}{lll}
0 & \text { if } & r=1 \\
a_{1} z+a_{3} z^{3}+\cdots+a_{r-2} z^{r-2} & \text { if } & r \neq 1
\end{array}\right.
$$

and

$$
\lambda(z)=\gamma(z)-z^{-r} P(z)
$$

Then (6.2) becomes,

$$
\begin{aligned}
& \frac{1}{2 \pi} \int_{0}^{2 \pi} L\left(t e^{i \theta}\right)(\cos \theta+\sin \theta) d \theta \\
& \quad=t^{r}(-1)^{m} \int_{0}^{\infty} S_{u}^{m+1} \frac{d^{m+2}}{d u^{m+2}}\left[(u t)^{-r} P(u t)+\lambda(u t)\right] d u
\end{aligned}
$$




$$
\begin{aligned}
= & t^{r}(-1)^{m} \int_{0}^{\infty} S_{u}^{m+1} \frac{d^{m+2}}{d u^{m+2}}\left[(u t)^{-r} P(u t)\right] d u \\
& +t^{r}(-1)^{m} \int_{0}^{\infty} S_{u}^{m+1} \frac{d^{m+2}}{d u^{m+2}} \lambda(u t) d u \\
= & A(t)+t^{r} B(t) . \\
A= & t^{r}(-1)^{m} \int_{1 / 2}^{\infty} S_{u}^{m+1} \frac{d^{m+2}}{d u^{m+2}}\left(\sum_{k=1}^{r-2} a_{k}(u t)^{k-r}\right) d u \\
= & \sum_{\substack{k=1 \\
k \text { odd }}}^{r-2} t^{r+k-r} a_{k}(-1)^{m} \int_{1 / 2}^{\infty} o(u)^{m+1} \frac{d^{m+2}}{d u^{m+2}} u^{k-r} d u \\
= & \sum_{\substack{k=1 \\
k \text { odd }}}^{r-2} b_{k} t^{k} .
\end{aligned}
$$

Hence,

$$
\frac{1}{2 \pi} \int_{0}^{2 \pi} L\left(t e^{i \theta}\right)(\cos \theta+\sin \theta) d \theta=\sum_{\substack{k=1 \\ k \text { odd }}}^{r-2} b_{k} t^{k}+t^{r} B(t)
$$

where

$$
B(t)=(-1)^{m} \int_{0}^{\infty} S_{u}^{m+1} \frac{d^{m+2}}{d u^{m+2}} \lambda(u t) d u
$$

To complete the proof of Theorem 2 we must show $B(t) \rightarrow 0$ as $t \rightarrow 0$. If $f(u)$ is a function defined for $u>0$ and $\eta$ is a positive real number, denote by

$$
I^{\eta} f(z)=\frac{1}{\Gamma(\eta)} \int_{0}^{z}(z-u)^{\eta-1} f(u) d u
$$

the fractional integral of order $\eta$, see [6]. Now if we set

$$
f(u)=S_{u}=\sum_{|n|<u} c_{n},
$$

then by (4.1),

$$
S_{u}^{\eta}=I^{\eta} S_{u},
$$

so

$$
\begin{aligned}
S_{u}^{m+1} & =I^{m+1} S_{u} \\
& =I^{1-\alpha} I^{m+\alpha} S_{u} \\
& =I^{1-\alpha} S_{u}^{m+\alpha}
\end{aligned}
$$

Thus,

$$
\begin{aligned}
S_{u}^{m+1} & =\frac{1}{\Gamma(1-\alpha)} \int_{0}^{u}(u-z)^{1-\alpha-1} S_{z}^{m+\alpha} d z \\
& =\frac{1}{\Gamma(1-\alpha)} \int_{0}^{u}(u-z)^{-\alpha} S_{z}^{m+\alpha} d z
\end{aligned}
$$


Returning to (6.4)

$$
\begin{aligned}
B(t) & =(-1)^{m} \int_{0}^{\infty} S_{u}^{m+1} \frac{d^{m+2}}{d u^{m+2}} \lambda(u t) d u \\
& =\lim _{R \rightarrow \infty}(-1)^{m} \int_{0}^{R} \frac{1}{\Gamma(1-\alpha)} \int_{0}^{u}(u-z)^{-\alpha} S_{z}^{m+\alpha} d z \frac{d^{m+2}}{d u^{m+2}} \lambda(u t) d u \\
& =\lim _{R \rightarrow \infty} \frac{(-1)^{m}}{\Gamma(1-\alpha)} \int_{0}^{R} S_{z}^{m+\alpha} \int_{z}^{R}(u-z)^{-\alpha} \frac{d^{m+2}}{d u^{m+2}} \lambda(u t) d u d z \\
& =\lim _{R \rightarrow \infty} \frac{(-1)^{m}}{\Gamma(1-\alpha)} \int_{0}^{R} S_{z}^{m+\alpha} H(z, t, R) d z,
\end{aligned}
$$

where

$$
\begin{aligned}
& H(z, t, R)=\int_{z}^{R}(u-z)^{-\alpha} \frac{d^{m+2}}{d u^{m+2}} \lambda(u t) d u \\
& B(t)=\lim _{R \rightarrow \infty} \frac{(-1)^{m}}{\Gamma(1-\alpha)} \int_{0}^{1 / t} S_{z}^{m+\alpha} H(z, t, R) d z \\
& \quad+\lim _{R \rightarrow \infty} \frac{(-1)^{m}}{\Gamma(1-\alpha)} \int_{1 / t}^{R} S_{z}^{m+\alpha} H(z, t, R) d z \\
& =B_{1}(t)+B_{2}(t) .
\end{aligned}
$$

We will make separate estimates of $H(z, t, R)$ for $B_{1}(t)$ and for $B_{2}(t)$.

First, in the interval of integration involving $B_{1}(t), 0 \leqq z \leqq 1 / t$.

$$
\begin{aligned}
H(z, t, R) & =\int_{z}^{R}(u-z)^{-\alpha} \frac{d^{m+2}}{d u^{m+2}} \lambda(u t) d u \\
& =\int_{z}^{1 / t}+\int_{1 / t}^{R} \\
& =H_{1}+H_{2} .
\end{aligned}
$$

Using the fact that $\lambda$ is entire,

$$
\begin{aligned}
\left|H_{1}\right| & \leqq \int_{z}^{1 / t}(z-u)^{-\alpha} t^{m+2} \cdot K d u \\
& \leqq K t^{m+2} \int_{z}^{1 / t}(z-u)^{-\alpha} d u \\
& =O\left(t^{m+2}\right)\left(\frac{1}{t}-z\right)^{1-\alpha} .
\end{aligned}
$$

We estimate $H_{2}$ by employing (5.11)

$$
\begin{aligned}
H_{2} & =\int_{1 / t}^{R}(u-z)^{-\alpha} \frac{d^{m+2}}{d u^{m+2}} \lambda(u t) d u \\
& =\int_{1 / t}^{\infty}(u-z)^{-\alpha} t^{m+2} O(u t)^{-r-1 / 2} d u
\end{aligned}
$$




$$
\begin{aligned}
& =O\left(t^{m-r+3 / 2}\right)\left(\frac{1}{t}-z\right)^{-\alpha} \int_{1 / t}^{\infty} u^{-r-1 / 2} d u \\
& =O\left(t^{m-r+3 / 2}\right)\left(\frac{1}{t}-z\right)^{-\alpha}\left(\frac{1}{t}\right)^{-r+1 / 2} \\
& =O\left(t^{m+1}\right)\left(\frac{1}{t}-z\right)^{-\alpha} .
\end{aligned}
$$

Returning to (6.5),

$$
H(z, t, R)=O\left(t^{m+2}\right)\left(\frac{1}{t}-z\right)^{1-\alpha}+O\left(t^{m+1}\right)\left(\frac{1}{t}-z\right)^{-\alpha} .
$$

and

$$
\begin{aligned}
B_{1}(t) & =\frac{(-1)^{m}}{\Gamma(1-\alpha)} \int_{0}^{1 / t} S_{z}^{m+\alpha} H(z, t, R) d z \\
& =\int_{0}^{1 / t} o\left(z^{m+\alpha}\right)\left\{O\left(t^{m+2}\right)\left(\frac{1}{t}-z\right)^{1-\alpha}+O\left(t^{m+1}\right)\left(\frac{1}{t}-z\right)^{-\alpha}\right\} d z \\
& =o\left(\frac{1}{t}\right)^{m+\alpha}\left\{O\left(t^{m+2}\right) \int_{0}^{1 / t}\left(\frac{1}{t}-z\right)^{1-\alpha} d z+O\left(t^{m+1}\right) \int_{0}^{1 / t}\left(\frac{1}{t}-z\right)^{-\alpha} d z\right\} \\
& =o\left(\frac{1}{t}\right)^{m+\alpha}\left\{O\left(t^{m+2}\right)\left(\frac{1}{t}\right)^{2-\alpha}+O\left(t^{m+1}\right)\left(\frac{1}{t}\right)^{1-\alpha}\right\} \\
& =o(1),
\end{aligned}
$$

as $t \rightarrow 0$.

It remains to be shown that $B_{2}(t) \rightarrow 0$. In the interval of integration for $B_{2}, 1 / t \leqq z \leqq R$, and

$$
\begin{gathered}
H(z, t, R)=\int_{z}^{R}(u-z)^{-\alpha} \frac{d^{m+2}}{d u^{m+2}} \lambda(u t) d u \\
=\int_{z}^{R}(u-z)^{-\alpha} \frac{d^{m+2}}{d u^{m+2}}\left(\frac{-P(u t)}{(u t)^{r}}\right) d u \\
\quad+\int_{z}^{R}(u-z)^{-\alpha} \frac{d^{m+2}}{d u^{m+2}} \gamma(u t) d u \\
=H_{a}+H_{b} \cdot \\
H_{a}=-\int_{z}^{R}(u-z)^{-\alpha} \frac{d^{m+2}}{d u^{m+2}}\left(\sum_{k=1}^{r-2} a_{k}(u t)^{k-r}\right) d u \\
=\int_{z}^{R}(u-z)^{-\alpha} t^{m+2} O(u t)^{-m-4} d u \\
=t^{-2}\left\{\int_{z}^{2 z}(u-z)^{-\alpha} O(u)^{-m-4} d u+\int_{2 z}^{\infty}(u-z)^{-\alpha} O(u)^{-m-4} d u\right\} \\
=t^{-2}\left\{O(z)^{1-\alpha} z^{-m-4}+O\left(z^{-\alpha}\right) z^{-m-3}\right\} \\
=t^{-2} O\left(z^{-m-\alpha-3}\right) \cdot
\end{gathered}
$$


We change variables in the interval for $H_{b}$ by letting $x=u t$.

$$
\begin{aligned}
H_{b}(z, t, R) & =\int_{z}^{R}(u-z)^{-a} \frac{d^{m+2}}{d u^{m+2}} \gamma(u t) d u \\
& =\int_{t z}^{t R}\left(\frac{x}{t}-z\right)^{-a} t^{m+2} \frac{d^{m+2}}{d u^{m+2}} \gamma(x) \frac{d x}{t} \\
& =t^{m+1+\alpha} \int_{t z}^{t R}(x-t z)^{-\alpha} \gamma^{(m+2)}(x) d x \\
& =t^{m+1+\alpha}\left\{\int_{t z}^{t z+1}+\int_{t z+1}^{t R}\right\} \\
& =H_{b}^{\prime}+H_{b}^{\prime \prime} .
\end{aligned}
$$

Recall that $1 / t \leqq z$, so in the interval of integration for $H_{b}, x>t z \geqq 1$. Thus, by (5.11)

$$
\left|\gamma^{(m+2)}(x)\right| \leqq C x^{-r-1 / 2},
$$

and

$$
\begin{aligned}
H_{b}^{\prime} & =t^{m+1+\alpha} \int_{t z}^{t z+1}(x-t z)^{-\alpha} \gamma^{(m+2)}(x) d x \\
& =t^{m+1+\alpha} O(t z)^{-r-1 / 2} \int_{t z}^{t z+1}(x-t z)^{-\alpha} d x \\
& =t^{m+1+\alpha} O(t z)^{-r-1 / 2} .
\end{aligned}
$$

We estimate $H_{b}^{\prime \prime}$ by integrating by parts.

$$
\begin{aligned}
H_{b}^{\prime \prime}= & t^{m+1+\alpha} \int_{t z+1}^{t R}(x-t z)^{-\alpha} \gamma^{(m+2)}(x) d x \\
= & \left.t^{m+1+\alpha}(x-t z)^{-\alpha} \gamma^{\prime m+1)}(x)\right|_{t z+1} ^{t R} \\
& +t^{m+1+\alpha} \alpha \int_{t z+1}^{t R}(x-t z)^{-\alpha-1} \gamma^{(m+1)}(x) d x \\
= & \left.t^{m+1+\alpha}(x-t z)^{-\alpha} \gamma^{(m+1)}(x)\right|_{t z+1} ^{t R} \\
& +t^{m+1+\alpha} O(t z)^{-r-1 / 2} \int_{t z+1}^{t R}(x-t z)^{-\alpha-1} d x \\
= & t^{m+1+\alpha}(t R-t z)^{-\alpha} \gamma^{(m+1)}(t R)-t^{m+1+\alpha} \gamma^{(m+1)}(t z+1) \\
& +t^{m+1+\alpha} O(t z)^{-r-1 / 2}\left(\frac{1}{-\alpha}\right)\left\{(t R-t z)^{-\alpha}-1\right\} \\
= & t^{m+1+\alpha}(t R-t z)^{-\alpha} O(t z)^{-r-1 / 2}+t^{m+1+\alpha} O(t z)^{-r-1 / 2} \\
= & t^{m+1+\alpha} O(t z)^{-r-1 / 2} .
\end{aligned}
$$

Hence, in the interval of integration for $B_{2}$,

$$
\begin{aligned}
H_{b}(z, t, R) & =H_{b}^{\prime}+H_{b}^{\prime \prime} \\
& =t^{m+1+\alpha} O(t z)^{-r-1 / 2},
\end{aligned}
$$


and

$$
\begin{aligned}
H(z, t, R) & =H_{a}+H_{b} \\
& =t^{-2} O\left(z^{-m-\alpha-3}\right)+t^{m+1+\alpha} O(t z)^{-r-1 / 2} .
\end{aligned}
$$

So,

$$
\begin{aligned}
B_{2}(t) & =\lim _{R \rightarrow \infty} \frac{(-1)^{m}}{\Gamma(1-\alpha)} \int_{1 / t}^{R} S_{z}^{m+\alpha} H(z, t, R) d z \\
& =\lim _{R \rightarrow \infty} \frac{(-1)^{m}}{\Gamma(1-\alpha)} \int_{1 / t}^{R} o(z)^{m+\alpha}\left\{t^{-2} O\left(z^{-m-\alpha-3}\right)+t^{m+1+\alpha} O(t z)^{-r-1 / 2}\right\} d z \\
& =t^{-2} \int_{1 / t}^{\infty} o\left(z^{m+\alpha-m-\alpha-3}\right) d z+t^{m+1+\alpha-r-1 / 2} \int_{1 / t}^{\infty} o\left(z^{m+\alpha-r-1 / 2}\right) d z \\
& =\left.t^{-2} o\left(z^{-2}\right)\right|_{1 / t} ^{\infty}+\left.t^{m+1 / 2+\alpha-r} O\left(z^{m+\alpha-r+1 / 2}\right)\right|_{1 / t} ^{\infty} \\
& =o(1) .
\end{aligned}
$$

(Note that the hypothesis $\alpha<1 / 2$ is necessary here to insure that the last integral converge.) This completes the proof of Theorem 2.

\section{REFERENCES}

1. Erdelyi et al, Higher Transcendental Functions, vol. II, McGraw-Hill, New York, 1953.

2. G. H. Hardy, The second theorem of consistency for summable series, Proc. London Math. Soc., (2), 15 (1916), 72-88.

3. M. Kohn, Spherical convergence and integrability of multiple trigonometric series on hypersurfaces, Studia Math., 44 (1972), 345-354.

4. M. Kohn, Riemann summability of multiple trigonometric series, Indiana Univ. Math. J., 24 (1975), 813-823.

5. M. Kohn, Lebesgue summability of double trigonometric series, Trans. Amer. Math. Soc., to appear.

6. M. Riesz, L'integrale de Riemann-Liouville et le problem de Cauchy, Acta Math., 81 (1949), 1-233.

7. V. Shapiro, Circular summability $C$ of double trigonometric series, Trans. Amer. Math. Soc., 76 (1954), 223-233.

8. A. Zygmund, Trigonometric Series, Vol. II, Cambridge University Press, Cambridge, 1968.

Received March 30, 1976 and in revised form October 6, 1976. Research partially sponsored by the City University of New York Research Foundation.

Brooklyn College of the City UNIVERSity of NeW YoRK

BROOKLYN, NY 11210 


\section{PACIFIC JOURNAL OF MATHEMATICS}

\section{EDITORS}

RICHARD ARENS (Managing Editor)

University of California

Los Angeles, CA 90024

R. A. BEAUMONT

University of Washington

Seattle, WA 98105

C. C. Moore

University of California

Berkeley, CA 94720

\section{J. DugundJI}

Department of Mathematics

University of Southern California

Los Angeles, CA 90007

R. Finn and J. Milgram

Stanford University

Stanford, CA 94305

\section{ASSOCIATE EDITORS}

\section{E. F. BECKENBACH}

B. H. NEUMANN

F. WoLF

K. YOSHIDA

\section{SUPPORTING INSTITUTIONS}

\author{
UNIVERSITY OF BRITISH COLUMBIA \\ CALIFORNIA INSTITUTE OF TECHNOLOGY \\ UNIVERSITY OF CALIFORNIA \\ MONTANA STATE UNIVERSITY \\ UNIVERSITY OF NEVADA \\ NEW MEXICO STATE UNIVERSITY \\ OREGON STATE UNIVERSITY \\ UNIVERSITY OF OREGON \\ OSAKA UNIVERSITY
}

\author{
UNIVERSITY OF SOUTHERN CALIFORNIA \\ STANFORD UNIVERSITY \\ UNIVERSITY OF HAWAII \\ UNIVERSITY OF TOKYO \\ UNIVERSITY OF UTAH \\ WASHINGTON STATE UNIVERSITY \\ UNIVERSITY OF WASHINGTON \\ AMERICAN MATHEMATICAL SOCIETY
}

The Supporting Institutions listed above contribute to the cost of publication of this Journal, but they are not owners or publishers and have no responsibility for its content or policies.

Mathematical papers intended for publication in the Pacific Jaurnal of Mathematics should be in typed form or offset-reproduced, (not dittoed), double spaced with large margins. Please do not use built up fractions in the text of your manuscript. You may however, use them in the displayed equations. Underline Greek letters in red, German in green, and script in blue. The first paragraph or two must be capable of being used separately as a synopsis of the entire paper. Items of the bibliography should not be cited there unless absolutely necessary, in which case they must be identified by author and Journal, rather than by item number. Manuscripts, in triplicate, may be sent to any one of the editors. Please classify according to the scheme of Math. Reviews, Index to Vol. 39. All other communications should be addressed to the managing editor, or Elaine Barth, University of California, Los Angeles, California, 90024.

The Pacific Journal of Mathematics expects the author's institution to pay page charges, and reserves the right to delay publication for nonpayment of charges in case of financial emergency

100 reprints are provided free for each article, only if page charges have been substantially paid. Additional copies may be obtained at cost in multiples of 50 .

The Pacific Journal of Mathematics is issued monthly as of January 1966. Regular subscription rate: $\$ 7200$ a year (6 Vols., 12 issues). Special rate: $\$ 36.00$ a year to individual members of supporting institutions.

Subscriptions, orders for back numbers, and changes of address should be sent to Pacific Journal of Mathematics, 103 Highland Boulevard, Berkeley, California, 94708.

PUBLISHED BY PACIFIC JOURNAL OF MATHEMATICS, A NON-PROFIT CORPORATION

Printed at Kokusai Bunken Insatsusha (International Academic Printing Co., Ltd.). 8-8, 3-chome, Takadanobaba, Shinjuku-ku, Tokyo 160, Japan.

Copyrit (C) 1975 by Pacific Journal of Mathematics Manufactured and first issued in Japan 


\section{Pacific Journal of Mathematics \\ Vol. 69, No. $2 \quad$ June, 1977}

Carol Alf and Thomas Alfonso O'Connor, Unimodality of the Lévy spectral

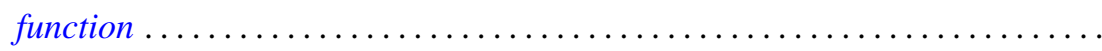

S. J. Bernau and Howard E. Lacey, Bicontractive projections and reordering of

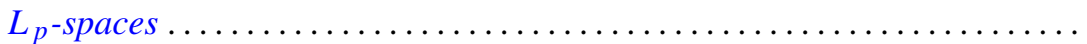

Andrew J. Berner, Products of compact spaces with bi-k and related spaces..... 303

Stephen Richard Bernfeld, The extendability and uniqueness of solutions of ordinary differential equations ...............................

Marilyn Breen, Decompositions for nonclosed planar m-convex sets ..........

Robert F. Brown, Cohomology of homomorphisms of Lie algebras and Lie

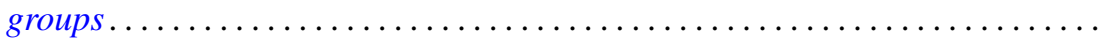

Jack Douglas Bryant and Thomas Francis McCabe, A note on Edelstein's iterative test and spaces of continuous functions ....................

Victor P. Camillo, Modules whose quotients have finite Goldie dimension ....... 333

David Downing and William A. Kirk, A generalization of Caristi's theorem with

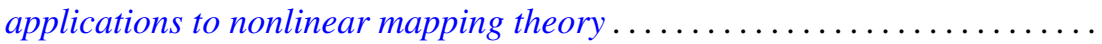

Daniel Reuven Farkas and Robert L. Snider, Noetherian fixed rings ...........

Alessandro Figà-Talamanca, Positive definite functions which vanish at

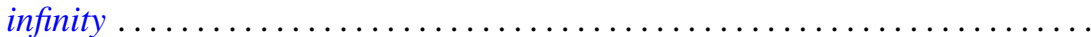

Josip Globevnik, The range of analytic extensions .................. 365

André Goldman, Mesures cylindriques, mesures vectorielles et questions de

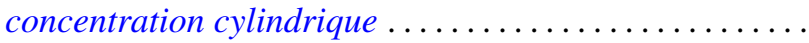

Richard Grassl, Multisectioned partitions of integers..........

Haruo Kitahara and Shinsuke Yorozu, A formula for the normal part of the

Laplace-Beltrami operator on the foliated manifold .... .

Marvin J. Kohn, Summability $R_{r}$ for double series .........

Charles Philip Lanski, Lie ideals and derivations in rings with involution ..

Solomon Leader, A topological characterization of Banach contractions . .

Daniel Francis Xavier O’Reilly, Cobordism classes of fiber bundles . .

James William Pendergrass, The Schur subgroup of the Brauer group . .

Howard Lewis Penn, Inner-outer factorization of functions whose Fourier series

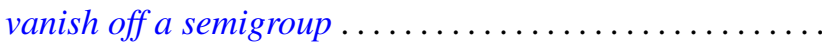

501

William T. Reid, Some results on the Floquet theory for disconjugate definite

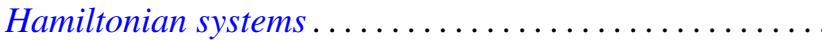

Caroll Vernon Riecke, Complementation in the lattice of convergence

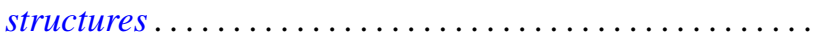

Louis Halle Rowen, Classes of rings torsion-free over their centers ......... 527

Manda Butchi Suryanarayana, A Sobolev space and a Darboux problem ....... 535

Charles Thomas Tucker, II, Riesz homomorphisms and positive linear maps.... 551

William W. Williams, Semigroups with identity on Peano continua ........... 557

Yukinobu Yajima, On spaces which have a closure-preserving cover by finite 